Nágila Soares Xavier Oenning ${ }^{1}$ Fernando Martins Carvalho ${ }^{2}$ Verônica Maria Cadena Lima ${ }^{3}$

\section{Indicadores de absenteísmo e diagnósticos associados às licenças médicas de trabalhadores da área de serviços de uma indústria de petróleo*}

\author{
Absenteeism indicators and diagnosis associated to sick \\ leave among workers of the administrative service area of a \\ petroleum industry
}

1 Enfermeira do Trabalho e Mestre em Saúde, Ambiente e Trabalho. Discente do Programa de Pós-Graduação em Saúde, Ambiente e Trabalho da Universidade Federal da Bahia, Salvador, BA, Brasil.

2 Pós-Doutorado em Epidemiologia pela University of Massachusetts at Lowell. Professor titular do Departamento de Medicina Preventiva e Social da Universidade Federal da Bahia, Salvador, BA, Brasil.

${ }^{3}$ Doutorado em Estatística pela University Leeds (Inglaterra). Professora adjunta do Departamento de Estatística da Universidade Federal da Bahia, Salvador, BA, Brasil.

*Trabalho baseado na dissertação de mestrado de Nágila Soares Xavier Oenning, intitulada Absenteísmo com licença médica em uma coorte de trabalhadores da área de serviços de uma indústria de petróleo, defendida em 2011 no Programa de Pós-Graduação em Saúde, Ambiente e Trabalho da Universidade Federal da Bahia (UFBA).

Não há conflitos de interesses neste trabalho e não houve financiamento para sua realização.

Trabalho apresentado e resumo publicado no VIII Congresso Brasileiro de Epidemiologia

Contato:

Nágila Soares Xavier Oenning

Avenida Carlos Gomes, 222, $7^{\circ}$ andar

Auxiliadora, Porto Alegre-RS

CEP: $90480-000$

E-mail:

nagilasx@gmail.com

Recebido: 14/10/2011

Revisado: 25/04/2012

Aprovado: 02/05/2012

\section{Resumo}

Objetivos: Determinar indicadores do absenteísmo por licença médica (LM) em trabalhadores da área de serviços de uma indústria de petróleo, identificando as patologias associadas. Métodos: Este estudo descreve resultados de estudo coorte retrospectivo com 782 trabalhadores de uma empresa de petróleo no período de $1^{\circ}$ de janeiro de 2007 a 31 de dezembro de 2009. Resultados: 542 trabalhadores tiveram eventos de faltas ao trabalho que geraram licença médica. Registrou-se 3,3 episódios de LM por trabalhador e 69,3\% dos trabalhadores tiveram pelo menos um episódio de LM. Os episódios de LM duraram em média 6,6 dias, com desvio padrão de 9,8 dias. A maior proporção de episódios de absenteísmo por LM deveu-se às doenças do sistema osteomuscular e tecido conjuntivo. Dezesseis dentre os 782 trabalhadores apresentaram 17 episódios de LM associadas ao trabalho (acidente típico, de trajeto e doença ocupacional). Conclusões: O estudo ratificou a importância do afastamento por doenças do sistema osteomuscular e o impacto das doenças ocupacionais no absenteísmo por licença médica, detectando índices semelhantes ao da literatura, numa população pouco explorada do ponto de vista epidemiológico.

Palavras-chave: absenteísmo; licença médica; trabalhadores; indústria petroquímica; saúde do trabalhador.

\begin{abstract}
Objective: To determine indices of sick leave (SL) absenteeism among workers in the administrative service area of a petroleum industry and to identify associated pathologies. Methods: The study describes results of a retrospective cohort study involving 782 workers from an oil company, who were followed up from January 1, 2007 to December 31, 2009. Results: During the study period, 542 workers reported SL events with physician's statements. An average of 3.3 SL absences per worker was recorded, and $69.3 \%$ of the workers had gone through at least one SL episode. SL episodes lasted in average for 6.6 days, with a standard deviation of 9.8 days. Most of SL absences were due to musculoskeletal and connective tissue disorders. Sixteen out of the 782 workers went through 17 work-related SL episodes (worksite accidents, commuting accidents and occupational illnesses). Conclusions: The study confirmed that musculoskeletal and connective tissue diseases are the most recurrent causes of sick leave absenteeism. The rates of absenteeism due to SL, in this population yet rarely investigated from the epidemiological point of view, were similar to those described in literature.
\end{abstract}

Keywords: absenteeism; sick leave; workers; petroleum industry; occupational health. 


\section{Introdução}

O absenteísmo, absentismo ou ausentismo é uma expressão utilizada para designar a falta do empregado ao trabalho. O absenteísmo é considerado como o período de ausência laboral que se aceita como atribuível a uma incapacidade do indivíduo, exceção feita para aquela derivada de gravidez normal ou prisão (ORGANIZACIÓN INTERNACIONAL DEL TRABAJO, 1989).

Como um fenômeno multicausal, o absenteísmo não pode ser totalmente explicado pela abordagem de uma de suas dimensões, porém estudos sobre uma das vertentes pode suscitar novas explorações. Dentre os tipos existentes na realidade brasileira, o absenteísmo médico é o mais abordado, talvez porque possua maior controle documental, fato atribuído à necessidade de apresentação de licença médica (LM).

A ausência ao trabalho por uma doença ou situação de saúde (gravidez) é validada com a apresentação de um atestado médico. Muitos estudos brasileiros se utilizaram de buscas documentais em arquivos médicos na tentativa de explicar o fenômeno absenteísmo (SANTOS; MATTOS, 2010; CUNHA; BLANK; BOING, 2009; SILVA; PINHEIRO; SAKURAI, 2008; REIS et al., 2003; GUIMARÃES; CASTRO, 2007).

Da evolução do conceito de Medicina do Trabalho para o pensar em Vigilância à Saúde do Trabalhador emergiu a necessidade da observação de eventos no ambiente de trabalho, a fim de traçar diagnósticos situacionais e garantir intervenções de promoção e prevenção da saúde do trabalhador. Diante desse cenário, observar o evento adoecimento associado à ausência ao trabalho, torna-se relevante para a Saúde do Trabalhador, bem como para a perspectiva administrativa.

A análise do absenteísmo deve observar o caráter multifatorial desse fenômeno, considerando variáveis diversas, tais como hábitos, valores, habilidades e conhecimentos; variáveis relacionadas à ocupação do trabalhador, como o tipo de empresa e os métodos de produção; variáveis relacionadas à organização, como o clima de trabalho e as políticas da instituição (SALDARRÍAGA; MARTÍNEZ, 2007).

Estudos (ORGANIZACIÓN INTERNACIONAL DEL TRABAJO, 1989; KREMER; STEENBEEK, 2010; FUNDAÇÃO EUROPEIA PARA A MELHORIA DAS CONDIÇÔES DE VIDA E DE TRABALHO, 1997) apontam que a maior parte do absenteísmo no trabalho é justificada pela LM. A LM justifica, além do adoecimento, fatores como maternidade, pessoa em boa saúde que acompanha pessoa doente e ausência para realização de exames de rotina.
Diante da necessidade de entender o cenário de absenteísmo na indústria, a questão norteadora foi: "qual o panorama do absenteísmo justificado por licença médica"? Espera-se que o entendimento deste fenômeno sirva como fomento para propostas mais assertivas de promoção da saúde do trabalhador.

O presente estudo objetivou: 1 - Determinar indicadores do absenteísmo por licença médica, ocupacional e não ocupacional em trabalhadores da área de serviços de uma indústria de petróleo; e 2 - Identificar as patologias associadas ao absenteísmo.

\section{Métodos}

Este artigo descreve resultados de um estudo de coorte retrospectiva realizado com todos os 782 trabalhadores de uma regional de uma empresa de petróleo no período de $1^{\circ}$ de janeiro de 2007 a 31 de dezembro de 2009. Os dados foram obtidos através do prontuário do trabalhador, no banco de dados (software SD2000*) do serviço de saúde ocupacional de uma empresa de petróleo do Brasil. A área estudada, realiza atividades de apoio ao processo fim da empresa: aquisição de bens e serviços, assessoria em Saúde, Meio Ambiente e Segurança (SMS), gestão de saúde suplementar, logística, recursos humanos e capacitação, administração de prédios, segurança patrimonial, despacho aduaneiro, montagem de escritório, dentre outros. A regional em estudo possui sede na cidade de Salvador-Bahia, com trabalhadores distribuídos pelos estados do Norte e Nordeste do Brasil. Seus serviços são executados em área administrativa (escritórios) e operacional (portos, refinarias e campos de exploração de petróleo).

A coorte foi composta por trabalhadores com vínculo direto via processo seletivo que estivessem presentes na folha de pagamento de 01/01/2007 (tempo zero). Havia 787 trabalhadores neste ponto do tempo. Destes, cinco foram excluídos porque se encontravam afastados do trabalho por doença (em LM) no tempo zero e permaneceram afastados durante todo o período do estudo e, consequentemente, sem nenhum dado de exame periódico nem informação de dias potenciais trabalháveis. Portanto, restaram 782 trabalhadores para o estudo. Durante o acompanhamento do estudo, 696 trabalhadores permaneceram todo o período e 86 foram perdidos de acompanhamento. Em 2007, houve 30 perdas; em 2008, 31 perdas e, em 2009, 25. As perdas aconteceram por óbito (4), aposentadoria (21), demissão (3) ou transferência do trabalhador (58).

A partir do banco de dados do serviço de saúde ocupacional da empresa, foram obtidos os afastamen-

${ }^{4}$ Informações sobre o software SD2000 disponível em: <http://www.sd2000.com.br/main/sd2000/default.aspx>. Acesso em: 30 maio 2012. 
tos por LM que tiveram início no período de 2007 a 2009. Foram consideradas as licenças homologadas pelo médico do trabalho ou odontólogo inseridas no banco de dados. Ainda foram inclusas no estudo as licenças maternidade, pois a gravidez em um ambiente corporativo só é considerada para fins de afastamento legal se houver uma licença médica.

As características demográficas consideradas neste estudo foram: sexo, idade, cargo, regime de trabalho e tempo de atuação. De cada LM, foram obtidas as seguintes variáveis: data de início, número de dias da licença, grande grupo da Classificação Internacional de Doenças - 10 ${ }^{\mathrm{a}}$ Revisão - CID-10 (ORGANIZAÇÃO MUNDIAL DA SAÚDE, 1995) e agrupamentos diagnósticos de acordo com lista de tabulação de morbidade dessa classificação.

A partir desses dados, foram apurados o número e a duração dos episódios de licenças e calculados os indicadores propostos pela Permanent Commission and International Association on Occupational Health (1973): FLM - frequência de LM (número de episódios de LM no período / total de trabalhadores no período); FT - frequência de trabalhadores com LM (número de trabalhadores com LM no período / total de trabalhadores no período); e, ainda, o indicador proposto por Hensing et al. (1998), IDA - índice de duração do absenteísmo (número total de dias de LM / número de episódios de LM).

Os cargos foram agrupados em três categorias, a saber: Técnico em Administração e Controle, Inspetor de Segurança Interna e Outros. Essa categorização deveu-se à representatividade de dois cargos que representam, juntos, 64,5\% da população.

As análises foram realizadas com os softwares Excel 2007 e SPSS versão 16.0. O estudo foi aprovado pelo Comitê de Ética e Pesquisa da Escola de Enfermagem da Universidade Federal da Bahia, protocolo $02 / 2010$.

\section{Resultados}

Os 782 trabalhadores tinham, no início da coorte, média de idade de 43,9 com desvio padrão (DP) de 8,5 anos e média de tempo de emprego na empresa de $14,9(\mathrm{DP}=9,8)$ anos. A população estudada era predominantemente do sexo masculino $(75,7 \%)$, na faixa de 41 a 50 anos de idade. O regime de trabalho administrativo ocupava 56,3\% dos trabalhadores e o de turno, $43,7 \%$. O cargo mais frequente foi o de Inspetor de Segurança Interna (43,6\%), seguido do de Técnico de Administração e Controle (20,8\% dos trabalhadores).

Em termos gerais, a população pode ser descrita como: não fumante $(74,2 \%)$, com sobrepeso $(44,1 \%)$, normotensa $(82,5 \%)$, com baixo risco para evento cardiovascular $(87,0 \%)$, sedentária $(80,0 \%)$, com sono normal $(84,7 \%)$ e glicemia até $100 \mathrm{mg} / \mathrm{dl}(83,9 \%)$. Os grupos com e sem LM não diferiram marcantemente quanto às características citadas acima.

Na população estudada, durante os três anos de observação, 542 trabalhadores tiveram eventos que geraram licença médica. Registraram-se 3,3 episódios de LM por trabalhador; FT de 0,693 - ou seja, $69,3 \%$ dos trabalhadores tiveram pelo menos um episódio de LM; e IDA de 6,6 - ou seja, os episódios de LM duraram em média 6,6 $(\mathrm{DP}=9,8)$ dias. O IDA diminuiu para 5,64 (DP $=7,5)$ dias após o expurgo das licenças maternidades.

A Tabela 1 mostra que dos 542 trabalhadores que se ausentaram durante o estudo, 130 (24,0\%) tiveram apenas um episódio de LM e 167 (30,8\%) se afastaram de duas a três vezes. Nota-se, ainda, que $63(11,6 \%)$ trabalhadores tiveram 10 ou mais licenças nos três anos. Dentre os 72 indivíduos com ausências maiores que 50 dias de duração, 27 $(37,5 \%)$ tiveram 10 ou mais episódios de LM. Por outro lado, dentre os 109 trabalhadores que apresentaram até 3 dias de ausência, 79 (60,8\%) tiveram apenas um episódio de LM.

As doenças ocupacionais apresentaram IDA elevado e FLM baixa, demonstrando seu caráter crônico-degenerativo. A licença maternidade apresentou IDA alto e FLM baixa, devido à predominância masculina na população. As doenças não ocupacionais apresentaram frequência maior de episódios de LM e baixo IDA, comparadas às outras nosologias (Tabela 2).

Os 542 trabalhadores faltosos somaram, no período, um total de 2564 episódios de ausência ao trabalho com licença médica. Em 18 (0,70\%) episódios, os dados estavam incompletos por não constar a nosologia ou o diagnóstico. Dos 2546 episódios com dados completos, 2447 (96,11\%) foram classificados pelos médicos do trabalho da empresa como doença não ocupacional com afastamento até 15 dias. Dezessete episódios $(0,66 \%)$ de faltas foram associados a fatores do trabalho. As três causas de afastamento mais incidentes foram: Doenças do sistema osteomuscular e do tecido conjuntivo (M00-M99), Doenças do aparelho digestivo (K00-K93) e Doenças do aparelho respiratório (J00-J99). O absenteísmo por licença maternidade representou apenas $0,43 \%$ do total de episódios (Tabela 3).

O detalhamento do IDA segundo os grandes grupos de classificação da CID-10 revela que as quatro principais causas que mantiveram o trabalhador afastado por mais tempo foram: Doenças do ouvido e da apófise mastoide (H60-H95), Transtornos mentais e comportamentais (F00-F99), Fatores que influenciam o estado de saúde e o contato com os serviços de saúde (Z00-Z99) e Lesões, envenenamento e algumas consequências de causas externas (S00-T98) (Tabela 4). Deve-se ressalvar que o grupamento dos códigos da CID (Z00-Z99) 
sofreu a influência dos 10 episódios de licença maternidade (Tabela 3), que totalizaram 1380 dias.

Para os grupos de doenças com maior frequência de episódios, o detalhamento dos códigos da CID-10 revelou que dentre as doenças do sistema osteomuscular e do tecido conjuntivo (M00-M99) os diagnósticos mais frequentes foram M54 - Dorsalgia e M65 - Sinovite e tenossinovite; dentre as doenças do aparelho digestivo (K00-K93), a maioria dos diagnósticos foi classificada nos subgrupos de causas odontológicas. Dentre as doenças do aparelho respiratório (J00-J99) os diagnósticos mais frequentes foram Influenza, Sinusite e Amigdalite (Tabela 5).

De 2007 a 2009, 16 (2,0\%) dentre os 782 trabalhadores da coorte apresentaram 17 episódios de LM associadas ao trabalho (acidente típico, doença ocupacional e acidente de trajeto), totalizando 1104 dias de ausência ao trabalho.

Dos 782 trabalhadores investigados houve emissão de Licença Médica em 4 (0,51\%) casos de acidente típico ocupacional. Todos os diagnósticos dos quatro episódios pertenciam ao grande grupo "Lesões, envenenamento e algumas outras consequências de causas externas (S00-T98)" da CID-10: Entorse e distensão do tornozelo, Ferimento do joelho, Ferimento na cabeça, parte não especificada, Fratura da extre- midade distal do rádio e do cúbito. Estes quatro trabalhadores faltaram 332 dias, com licença médica, e, desses, 59 foram devido aos episódios de acidente típico ocupacional.

Dos 782 trabalhadores, houve 5 (0,64\%) episódios de LM atribuídos à doença ocupacional. Todos os diagnósticos dos cinco episódios pertenciam ao grande grupo de doenças do sistema osteomuscular e do tecido conjuntivo (M00-M99) da CID-10: Cervicalgia, Sinovite e Tenossinovite, Outras Sinovites e Tenossinovites, Tendinite Bicipital, Síndrome do Manguito Rotador. Estes trabalhadores faltaram 837 dias de trabalho por licenças médicas com nosologia por doença ocupacional ou 76\% dos 1104 dos dias de ausência por causas associadas ao trabalho.

Três destes cinco trabalhadores com doença ocupacional não tiveram sua doença reconhecida como tal, na data da primeira licença médica, emitida durante o período do estudo. Por exemplo, um trabalhador teve como motivo da licença médica o diagnóstico de Outras Sinovites e Tenossinovites. Entretanto, o reconhecimento deste diagnóstico como doença ocupacional só veio a ocorrer após o décimo quinto episódio com diagnósticos iguais ou semelhantes. Ressalve-se que essas informações pertencem apenas ao período de seguimento da coorte.

Tabela 1 Episódios de licença médica segundo a duração das ausências, em trabalhadores de uma empresa de petróleo, 2007-2009

\begin{tabular}{|c|c|c|c|c|c|c|c|c|c|c|c|c|c|c|}
\hline \multirow{3}{*}{$\begin{array}{l}\text { Número de } \\
\text { episódios }\end{array}$} & \multicolumn{14}{|c|}{ Duração das ausências (dias) } \\
\hline & \multicolumn{2}{|c|}{ até 3} & \multicolumn{2}{|c|}{$4 a 10$} & \multicolumn{2}{|c|}{11 a 15} & \multicolumn{2}{|c|}{16 a 30} & \multicolumn{2}{|c|}{31 a 50} & \multicolumn{2}{|c|}{$>50$} & \multicolumn{2}{|c|}{ Total } \\
\hline & $n$ & $\%$ & $n$ & $\%$ & $n$ & $\%$ & $n$ & $\%$ & $n$ & $\%$ & $n$ & $\%$ & $n$ & $\%$ \\
\hline 1 & 79 & 60,8 & 29 & 22,3 & 17 & 13,1 & 2 & 1,5 & 0 & 0,0 & 3 & 2,3 & 130 & 100,0 \\
\hline 2 a 3 & 30 & 17,9 & 75 & 44,9 & 14 & 8,4 & 31 & 18,6 & 7 & 4,2 & 10 & 6,0 & 167 & 100,0 \\
\hline 4 a 9 & 0 & 0,0 & 29 & 15,9 & 28 & 15,4 & 66 & 36,3 & 27 & 14,8 & 32 & 17,6 & 182 & 100,0 \\
\hline 10 ou mais & 0 & 0,0 & 0 & 0,0 & 0 & 0,0 & 14 & 22,2 & 22 & 34,9 & 27 & 42,9 & 63 & 100,0 \\
\hline Total & 109 & 20,1 & 133 & 24,5 & 59 & 10,9 & 113 & 20,9 & 56 & 10,3 & 72 & 13,3 & 542 & 100,0 \\
\hline
\end{tabular}

Fonte: Banco de dados da Gerência de Segurança, Meio Ambiente e Saúde da empresa.

Tabela 2 Índice de duração do absenteísmo (IDA)* e frequência da licença médica (FLM)** segundo a nosologia do episódio, em trabalhadores de uma indústria do petróleo, 2007-2009

Nosologia do episódio

Índice de duração do absenteísmo

Frequência de licença médica

$\begin{array}{lcc}\text { Doença ocupacional } & 167,0 & 0,006 \\ \text { Licença maternidade } & 136,4 & 0,014 \\ \text { Acidente fora do trabalho } & 38,4 & 0,006 \\ \text { Acidente de trajeto } & 26,1 & 0,008 \\ \text { Acidente típico ocupacional } & 14,8 & 0,005 \\ \text { Doença não ocupacional } & 5,2 & 3,210\end{array}$

Fonte: Banco de dados da Gerência de Segurança, Meio Ambiente e Saúde da empresa.

*IDA: número total de dias de licença médica/número de episódios de licença médica.

*** FLM: número de episódios de licença médica no período/total de trabalhadores no período. 
Tabela 3 Diagnósticos associados às licenças médicas segundo a nosologia, em trabalhadores faltosos de uma indústria do petróleo, 2007-2009

\begin{tabular}{|c|c|c|c|c|c|c|c|c|c|c|c|}
\hline do & $\hat{\bar{N}}$ & $\stackrel{g}{=}$ & $\stackrel{\circ}{=}$ & $\hat{\infty}$ & $\stackrel{n}{\sim}$ & $\bar{\pi}$ & gे & : & $\stackrel{\circ}{\stackrel{+}{+}}$ & นี & ¿ \\
\hline $\bar{E}$ & กิ & 鬲 & 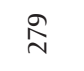 & $\overline{\mathrm{N}}$ & פ్ & $\stackrel{\infty}{-}$ & $\stackrel{n}{上}$ & $\stackrel{\tilde{n}}{\sim}$ & $\tilde{\varrho}$ & 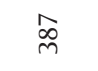 & 㞻 \\
\hline 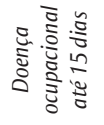 & - & 0 & 0 & 0 & 0 & 0 & 0 & 0 & 0 & 0 & - \\
\hline 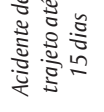 & 0 & 0 & 0 & 0 & 0 & 0 & in & 0 & 0 & 0 & in \\
\hline 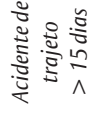 & 0 & 0 & 0 & 0 & 0 & 0 & $m$ & 0 & 0 & 0 & $m$ \\
\hline 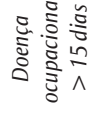 & 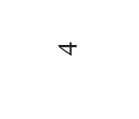 & 0 & 0 & 0 & 0 & 0 & 0 & 0 & 0 & 0 & 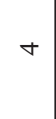 \\
\hline 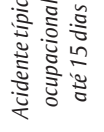 & 0 & 0 & 0 & 0 & 0 & 0 & $m$ & 0 & 0 & 0 & $m$ \\
\hline 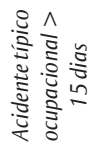 & 0 & 0 & 0 & 0 & 0 & 0 & - & 0 & 0 & 0 & - \\
\hline 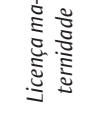 & 0 & 0 & 0 & 0 & 0 & 0 & 0 & 0 & 0 & $=$ & $=$ \\
\hline 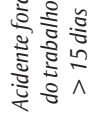 & 0 & 0 & 0 & 0 & 0 & 0 & $m$ & 0 & 0 & 0 & $m$ \\
\hline 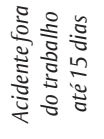 & - & 0 & 0 & 0 & 0 & 0 & - & 0 & 0 & 0 & N \\
\hline 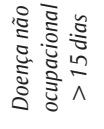 & $\stackrel{\operatorname{Ln}}{\sim}$ & in & 0 & - & - & 0 & 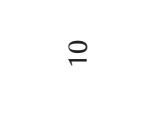 & $\sim$ & + & $\stackrel{\infty}{\sim}$ & $\Xi$ \\
\hline 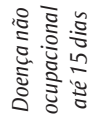 & $\tilde{\tilde{N}}$ & $\stackrel{\infty}{\sim}$ & $\stackrel{\curvearrowright}{\sim}$ & $\stackrel{\text { సి }}{ }$ & $\stackrel{\circ}{\circ}$ & $\stackrel{\infty}{\sim}$ & $\stackrel{g}{g}$ & in & g) & $\stackrel{\infty}{\text { \} }} &{\underset{\sim}{\stackrel{f}{*}}} \\
{\hline \text { 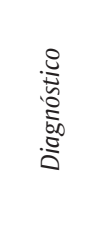 }} &{\text { 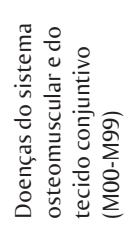 }} &{\text { 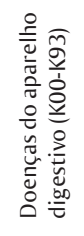 }} &{\text { 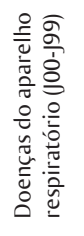 }} &{\text { 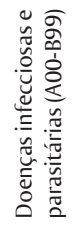 }} &{\text { 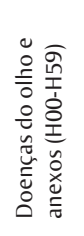 }} &{\text { 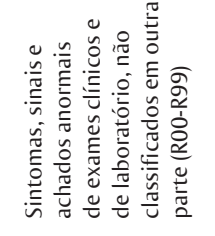 }} &{\text { 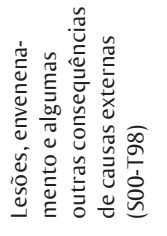 }} &{\text { 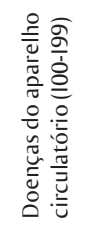 }} &{\text { 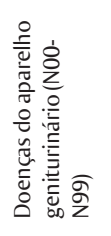 }} &{\text { 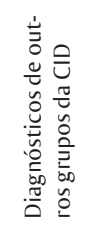 }} &{\begin{array}{l}\overline{\widetilde{J}} \\
\stackrel{\circ}{\circ}\end{array}} \\
$\hline
\end{tabular}


Tabela 4 Diagnósticos associados às licenças médicas em função dos dias de afastamento, números de episódios e índice de duração do absenteísmo (IDA)*, em trabalhadores de uma indústria do petróleo, 2007-2009

\begin{tabular}{|c|c|c|c|}
\hline Grande Grupo da CID-10 & Dias de absenteísmo & $N$ de episódios & $I D A$ \\
\hline Doenças do ouvido e da apófise mastoide (H60-H95) & 776 & 30 & 25,86 \\
\hline Transtornos mentais e comportamentais (F00-F99) & 1407 & 56 & 25,12 \\
\hline $\begin{array}{l}\text { Fatores que influenciam o estado de saúde e o contato com os serviços de } \\
\text { saúde (Z00-Z99) }\end{array}$ & 1717 & 93 & 18,46 \\
\hline $\begin{array}{l}\text { Lesões, envenenamento e algumas outras consequências de causas externas } \\
\text { (S00-T98) }\end{array}$ & 2106 & 175 & 12,03 \\
\hline Neoplasias (C00-D48) & 889 & 79 & 11,25 \\
\hline Gravidez, parto e puerpério (000-099) & 175 & 18 & 9,71 \\
\hline $\begin{array}{l}\text { Doenças do sangue e dos órgãos hematopoéticos e alguns transtornos } \\
\text { imunitários (D50-D89) }\end{array}$ & 6 & 1 & 6,36 \\
\hline $\begin{array}{l}\text { Doenças do sistema osteomuscular e do tecido conjuntivo } \\
\text { (M00-M99) }\end{array}$ & 3429 & 553 & 6,20 \\
\hline Doenças do aparelho circulatório (I00-199) & 916 & 153 & 5,98 \\
\hline Doenças do aparelho geniturinário (N00-N99) & 610 & 103 & 5,93 \\
\hline Doenças endócrinas, nutricionais e metabólicas (E00-E90) & 122 & 21 & 5,83 \\
\hline Doenças do olho e anexos (H00-H59) & 776 & 191 & 4,06 \\
\hline Doenças do sistema nervoso (G00-G99) & 92 & 23 & 4,01 \\
\hline Doenças do aparelho digestivo (К00-К93) & 1068 & 303 & 3,53 \\
\hline Doenças do aparelho respiratório (J00-J99) & 725 & 279 & 2,60 \\
\hline Doenças infecciosas e parasitárias (A00-B99) & 574 & 221 & 2,60 \\
\hline Doenças da pele e do tecido subcutâneo (L00-L99) & 159 & 63 & 2,52 \\
\hline $\begin{array}{l}\text { Sintomas, sinais e achados anormais de exames clínicos e de laboratório, não } \\
\text { classificados em outra parte (R00-R99) }\end{array}$ & 345 & 181 & 1,91 \\
\hline Causas externas de morbidade e de mortalidade (V01-Y98) & 5 & 3 & 1,59 \\
\hline Total & 15897 & 2546 & 6,24 \\
\hline
\end{tabular}

Fonte: Banco de dados da Gerência de Segurança, Meio Ambiente e Saúde da empresa.

* IDA: número total de dias de licença médica/número de episódios de licença médica. 
Tabela 5 Diagnósticos mais frequentes associados às licenças médicas conforme subgrupos da CID 10, entre trabalhadores de uma empresa de petróleo, 2007-2009

\begin{tabular}{lcc}
\hline \multicolumn{1}{c}{ Subgrupo da CID-10 e Diagnóstico } & ( & \\
& & \\
\hline Subgrupo da CID-10 (M00-M99) & 241 & 43,7 \\
M54 - Dorsalgia & 66 & 12,0 \\
M65 - Sinovite e tenossinovite & 245 & 44,3 \\
Outros diagnósticos da CID M00-M99 & 552 & 100,0 \\
Subtotal & & \\
Subgrupo da CID-10 (K00-K93) & 161 & 53,1 \\
Subgrupos de causas odontológicas (CID K00-K93) & 17 \\
K21 - Doença de refluxo gastresofágico & 125 & 41,3 \\
Outros diagnósticos da CID K09-K93 & 303 & 100,0 \\
Subtotal & & \\
Subgrupo da CID-10 (J00-J99) & & 100,0 \\
J11 - Influenza (gripe) devida a vírus não identificado & 49 & 17,6 \\
J01 - Sinusite aguda & 40 & 14,3 \\
J03 - Amigdalite aguda & 34 & 12,2 \\
J00 - Nasofaringite aguda (resfriado comum) & 31 \\
Outros diagnósticos da CID J00-J99 & 125 & \\
Subtotal & 279 & \\
\hline
\end{tabular}

Fonte: Banco de dados da Gerência de Segurança, Meio Ambiente e Saúde da empresa.

Dos 782 trabalhadores, 7 tiveram 8 episódios de LM por acidente de trajeto. Todos os diagnósticos dos oito episódios pertencem ao grande grupo Lesões, envenenamento e algumas outras consequências de causas externas (S00-T98) da CID-10: Contusão do joelho, Ferimento da pálpebra e da região periocular, Entorse e distensão do tornozelo, Contusão de dedo(s) com lesão da unha, Fratura de ossos do metatarso. Os trabalhadores que tiveram episódios de falta ao trabalho com LM por acidente de trajeto faltaram a 419 dias de trabalho e, desses, 208 foram devido aos episódios de acidente de trajeto.

\section{Discussão}

Este estudo descreve o absenteísmo por licença médica (LM) considerando a frequência dos episódios, sua duração e os diagnósticos mais frequentemente relacionados ao trabalho, dentre outros aspectos, mantendo o caráter epidemiológico, sem prejuízos para o trabalhador, conforme recomendação da Organização Internacional do Trabalho OIT (1985).
Características demográficas semelhantes aos participantes deste estudo foram observadas em uma pesquisa sobre absenteísmo em bancários (SILVA; PINHEIRO; SAKURAI, 2008). O grupo da empresa de petróleo aqui estudado caracterizou-se pela predominância de trabalhadores do sexo masculino, que obtiveram maior número de licenças médicas homologadas do que o sexo feminino.

A frequência de trabalhadores que tiveram pelo menos um episódio de LM - FT (69,3\%) supera em aproximadamente $20 \%$ das frequências relatadas em estudos nacionais e internacionais (SANTOS; MATTOS, 2010; KREMER; STEENBEEK, 2010). Em um estudo em profissionais de enfermagem, Silva e Marziale (2000) relatam uma FT maior que a do atual estudo. Os estudos descritivos sobre absenteísmo raramente abordam fatores relacionados à saúde, e sim as características das LMs. Aqueles que abordam características de saúde são analíticos e abordam, em sua maioria, somente uma característica associada ao absenteísmo.

Observou-se que $24,0 \%$ dos indivíduos que faltaram tiveram apenas 1 episódio de LM e que 54,8\% 
desses indivíduos tiveram até 3 episódios. Este comportamento revela que mais da metade dos indivíduos não obtém LM com alta recorrência. Um estudo em profissionais de enfermagem relatou a ocorrência de apenas um episódio em $37,6 \%$ da população investigada (FERRIE et al., 2009).

Os resultados deste estudo permitem concluir que quanto maior o número de episódios de LM maior a duração da ausência (Tabela 1).

A maior frequência de episódios de absenteísmo por LM estava relacionada às doenças do sistema osteomuscular e do tecido conjuntivo, tendência corroborada por diversos estudos (KREMER; STEENBEEK, 2010; SILVA; PINHEIRO; SAKURAI, 2008, REIS et al., 2003; FERRIE et al., 2009; CUNHA; BLANK; BOING, 2009; ALVES; GODOY; SANTANA, 2006; KNUTSSON; GOINE, 1998; PAWLINA; CAMPOS; RIBEIRO, 2009). Em seguida, apareceram as doenças do aparelho digestivo e doenças do aparelho respiratório. Quanto à gravidade do episódio, ou seja, quando ocorreu a combinação de baixa incidência de episódios com IDA alto, destacaram-se as doenças do ouvido e da apófise mastoide. Ressalta-se que, na literatura existente, não houve relato de magnitude desse tipo de diagnóstico. Logo em seguida, apareceram os transtornos mentais e comporta mentais, cuja magnitude é ratificada em diversos estudos (KREMER; STEENBEEK, 2010; SANTOS; MATTOS, 2010; SILVA; PINHEIRO; SAKURAI, 2008; FERRIE et al., 2009; CUNHA; BLANK; BOING, 2009; KNUTSSON; GOINE, 1998; PAWLINA; CAMPOS; RIBEIRO, 2009).

O absenteísmo estava associado aos fatores que influenciam o estado de saúde e o contato com os serviços de saúde, como já observado em outros estudos (CUNHA; BLANK; BOING, 2009; PAWLINA; CAMPOS; RIBEIRO, 2009; ALVES; GODOY; SANTANA, 2006). Dentre esses, encontram-se as licenças maternidades e as ausências para acompanhamento de familiar doente. Na análise desse estudo, foi observado que as ausências para acompanhamento de familiar doente não tiveram grande impacto neste grupo da CID, pois houve apenas dois episódios, somando dois dias de ausência. Já a licença maternidade, como esperado, contribuiu significativamente com o número de dias de ausências, apesar do baixo número de episódios. No período em estudo houve licenças de 120 dias, mas já foram contemplados episódios de 180 dias.

Dentre os trabalhadores faltosos, as doenças mais frequentes foram: dorsalgia, sinovites e tenossinovite, causas odontológicas e influenza. Daí infere-se a importância do investimento em programas de promoção da saúde e prevenção do adoecimento, com destaque para intervenções ergonômicas, de imunização, programas de saúde bucal e higiene industrial.
As doenças osteomusculares têm sido associadas ao absenteísmo em diversos estudos analíticos (DIAZ-LEDEZMA et al., 2009; ALEXOPOULOS et al., 2008; LOTTERS; BURDORF, 2006). Na América Latina foram encontrados resultados em que a dor lombar baixa representa 5,4\% de todo o absenteísmo e que o trabalhador com esse diagnóstico apresenta afastamento mais duradouro, quando comparado com o restante da população (DIAZ-LEDEZMA et al., 2009). Na Europa, o absenteísmo foi associado à dor lombar baixa (ALEXOPOULOS et al., 2008) e às doenças osteomusculares do ombro e extremidades superiores (LOTTERS; BURDORF, 2006).

Convém destacar que todos os casos de doença ocupacional deste estudo foram de doenças do sistema osteomuscular e que a duração desses episódios foi maior que os demais (Tabela 4). Observou-se, também, que esses episódios, em sua maioria, foram precedidos de LM pelo mesmo diagnóstico ou equivalente, porém com nosologia de doença não ocupacional.

Os acidentes de trabalho (típico e de trajeto) estavam representados pelos diagnósticos referentes às lesões, envenenamento e algumas outras consequências de causas externas (S00-T98), enquanto que as doenças ocupacionais estavam representadas pelos diagnósticos das doenças osteomusculares e do tecido conjuntivo (M00-M99), traduzindo os seus aspectos agudo e crônico, respectivamente.

No perfil de causas de absenteísmo por LM destacaram-se as doenças de causas odontológicas (Tabela 5). A empresa possui iniciativas de promoção da saúde bucal e fornece assistência odontológica suplementar. Porém, mesmo em empresas que executam todas as ações preventivas, detecta-se alto índice de afastamentos por motivos odontológicos (MELLO, 2006). Nesta população, a influenza ocupou lugar de destaque como causa de absenteísmo com LM, à semelhança do observado em outras populações (KREMER; STEENBEEK, 2010).

Observou-se a demora no reconhecimento de nosologias do sistema osteomuscular e do tecido conjuntivo como sendo doenças ocupacionais. Isso pode refletir a dificuldade de caracterização do evento doença ocupacional que geralmente é um fenômeno crônico, frequentemente de evolução lenta, que nem sempre deixa transparecer sua ligação com as atividades desempenhadas pelo trabalhador.

Concluindo, o estudo ratificou a importância do afastamento por doenças do sistema osteomuscular e o impacto das doenças ocupacionais no ausentismo por doença, detectando índices de absenteísmo semelhantes ao da literatura, numa população ainda pouco explorada do ponto de vista epidemiológico. 


\section{Contribuições de autoria}

OENNING, N. S. X.: realizou o delineamento e o planejamento do manuscrito, a coleta e a análise dos dados e participou da elaboração, da revisão e da versão final do manuscrito. CARVALHO, F. M.: participou da concepção do estudo, da análise dos dados, da elaboração, da revisão e da versão final do manuscrito. LIMA, V. M. C.: participou da análise dos dados, da elaboração, da revisão e da versão final do manuscrito.

\section{Referências}

ALEXOPOULOS, E. C. et al. Risk factors for sickness absence due to low back pain and prognostic factors for return to work in a cohort of shipyard workers. European Spine Journal, Heidelberg, v. 17, n. 9, p. 1185-1192, 2008.

ALVES, M.; GODOY, S. C. B.; SANTANA, D. M. Motivos de licenças médicas em um hospital de urgência-emergência. Revista Brasileira de Enfermagem, Brasília, v. 59, n. 2, p. 195-200, 2006.

CUNHA, J. B.; BLANK, V. L. G.; BOING, A. F. Tendência temporal de afastamento do trabalho em servidores públicos (1995-2005). Revista Brasileira de Epidemiologia, São Paulo, v. 12, n. 2, p. 226-236, 2009.

DIAZ-LEDEZMA, C. et al. Factors associated with variability in length of sick leave because of acute low back pain in Chile. Spine, New York, v. 9, n. 12, p. 1010-1015, 2009.

FERRIE, J. E. et al. Diagnosis-specific sickness absence and all-cause mortality in the GAZEL study. Journal of Epidemiology and Community Health, London, v. 63, n. 1, p. 50-55, 2009.

FUNDAÇÃO EUROPEIA PARA A MELHORIA DAS CONDIÇÕES DE VIDA E DE TRABALHO. A prevenção do absentismo no Trabalho. Luxemburgo: Serviço das Publicações Oficiais das Comunidades Europeias, 1997.

GUIMARÃES, R. S. O; CASTRO, H. A. O absenteísmo entre os servidores civis de um hospital militar. Revista Pesquisa Naval, Brasília, n. 20, p. 74-78, 2007.

HENSING, G. et al. How to measure sickness absence? Literature review and suggestion of five basic measures. Scandinavian Journal of Social Medicine, Stockholm, v. 26, n. 2, p. 133-144, 1998.

KNUTSSON, A.; GOINE, H. Occupation and unemployment rates as predictors of long term sickness absence in two Swedish counties. Social Science \& Medicine, New York, v. 47, n. 1, p. 25-31, 1998.

KREMER, A. M.; STEENBEEK, R. Avoidable sickness absence in a Dutch working population. Journal of Occupational Rehabilitation, New York, v. 20, n. 81, p. 81-89, 2010.

LOTTERS, F; BURDORF, A. Prognostic factors for duration of sickness absence due to musculoskeletal disorders. Clinical Journal of Pain, Philadelphia, v. 22, n. 2, p. 212-221, 2006.
MELLO, P. B. M. Odontologia do trabalho: uma visão multidisciplinar. Rio de Janeiro: Rubio, 2006.

ORGANIZAÇÃO INTERNACIONAL DO TRABALHO. Recomendação R171 - Serviços de Saúde Ocupacional. Genebra: OIT, 1985.

ORGANIZACIÓN INTERNACIONAL DEL TRABAJO. Absentismo: causa y control. In: ___. Enciclopedia de Salud y Seguridad en el Trabajo. Madrid: OIT, 1989. v.1, p. 5-12.

ORGANIZAÇÃO MUNDIAL DA SAÚDE. Centro Colaborador da OMS para a Classificação de Doenças em Português. Classificação estatística internacional de doenças e problemas relacionados à saúde. 10. revisão. São Paulo: Edusp, 1995.

PAWLINA, M. M. C.; CAMPOS, A. F; RIBEIRO, L. S. Características de absenteísmo entre trabalhadores da saúde: nível central da Secretaria de Estado de Saúde/ MT de 2005 a 2006. Revista Planejamento e Políticas Públicas, Brasília, n. 33, p. 173-194, 2009.

PERMANENT COMMISSION AND INTERNATIONAL ASSOCIATION ON OCCUPATIONAL HEALTH. Subcommittee on absenteeism: draft recommendations. British Journal of Industrial Medicine, London, v. 30, $n$. 4, p. 402-403, 1973.

REIS, R. J. et al. Fatores relacionados ao absenteísmo por doença em profissionais de enfermagem. Revista de Saúde Pública, São Paulo, v. 37, n. 5, p. 616-623, 2003.

SALDARRÍAGA, J. F.; MARTÍNEZ, E. Factores asociados al ausentismo laboral por causa médica en una institución de educación superior. Revista Facultad Nacional de Salud Pública, Medellin, v. 25, n. 1, p. 32-39, 2007.

SANTOS, J. P.; MATTOS, A. P. Absentismo-doença na prefeitura municipal de Porto Alegre, Rio Grande do Sul, Brasil. Revista Brasileira de Saúde Ocupacional, São Paulo, v. 35, n. 121, p. 148-156, 2010.

SILVA, D. M. P. P.; MARZIALE, M. H. P. Absenteísmo de trabalhadores de enfermagem em um hospital universitário. Revista Latino-Americana de Enfermagem, Ribeirão Preto, v. 8, n. 5, p. 44-51, 2000.

SILVA, L. S.; PINHEIRO, T. M. M.; SAKURAI, E. Perfil do absenteísmo em um banco estatal em Minas Gerais: análise no período de 1998 a 2003. Ciência \& Saúde Coletiva, Rio de Janeiro, v. 13, n. 2, p. 2049-2058, 2008. 